\title{
Multiple dosage forms of the NNRTI microbicide dapivirine: product development and evaluation Joseph Romano*
}

Address: International Partnership for Microbicides, Silver Spring, Maryland, USA

* Corresponding author

from 2006 International Meeting of The Institute of Human Virology

Baltimore, USA. 17-21 November, 2006

Published: 21 December 2006

Retrovirology 2006, 3(Suppl I):S54 doi:I0.II86/I742-4690-3-SI-S54

(C) 2006 Romano; licensee BioMed Central Ltd.

The disproportionate impact of the expanding AIDS epidemic in women of the developing world clearly establishes the need for anti-HIV microbicides. Although semisolid gels were the conventional dosage form developed for early microbicides, newer acceptability data and studies from the contraceptive field have demonstrated the need for additional alternative dosage forms that are acceptable to the relevant target populations. Reducing the burden of user compliance is equally critical in the next generation of products. Our development efforts with the highly potent NNRTI microbicide dapivirine have involved multiple dosage forms, with the objective of decoupling product use from sexual intercourse. Once per day gels and tablets are being developed, as are intravaginal ring delivery systems for sustained release. Preclinical development studies, formulation development and characterization, and clinical evaluations of these alternative drug product forms will be discussed. Our findings suggest that potent anti-retroviral compounds can be safely configured for acceptable dosing in women, and that these drugs and delivery strategies can be successfully applied in microbicide development. 